\title{
Lidocaine $5 \%$ patch for localized neuropathic pain: progress for the patient, a new approach for the physician
}

This article was published in the following Dove Press journal:

Clinical Pharmacology:Advances and Applications

29 March 2010

Number of times this article has been viewed

\author{
Guy Hans ${ }^{1,2}$ \\ Dominique Robert ${ }^{3}$ \\ Johanna Verhulst ${ }^{2}$ \\ Marcel Vercauteren ${ }^{1,2}$ \\ 'Department of Anesthesiology, \\ ${ }^{2}$ Multidisciplinary Pain Center, \\ ${ }^{3}$ Department of Intensive Care, \\ Antwerp University Hospital, \\ Edegem, Belgium
}

\begin{abstract}
Neuropathic pain ( $\mathrm{NeP}$ ) syndromes remain a difficult-to-treat medical entity. Despite a growing number of pharmacological and invasive analgesic therapies the results remain less than optimal because of insufficient analgesic efficacy and/or occurrence of pronounced side effects. Current guidelines propose the use of multimodal and balanced pharmacological therapies, focused on the underlying pathophysiological mechanisms (mechanistic approach). Lidocaine $5 \%$ patches are a new treatment option currently licensed for the treatment of postherpetic neuralgia. However, these patches can also be used for the treatment of different types of superficial NeP syndromes, such as diabetic polyneuropathy. Their therapeutic success, however, largely depends on the correct identification of appropriate patients and pain syndromes. This manuscript outlines the correct identification of patients and proper use of these patches in order to ensure as much as possible the therapeutic efficacy of this new treatment option.
\end{abstract}

Keywords: neuropathic pain, lidocaine, patch

\section{Introduction}

Despite considerable progress in the treatment options for neuropathic pain $(\mathrm{NeP})$ these conditions still remain very difficult to treat. ${ }^{1} \mathrm{NeP}$ remains a clinical challenge for several reasons, one of course being the specific pathophysiological features that underlie the occurrence of a painful neuropathic syndrome. A growing body of evidence indicates the presence of specific and multifactor alterations at several levels of the nervous system during $\mathrm{NeP}$ syndromes. ${ }^{2-6}$ Additionally, $\mathrm{NeP}$ patients often display co-morbidities that render the use of (strong) analgesics and/or additive drugs (such as anti-depressants and anti-epileptic agents) extremely difficult. This often leads to a trial-and-error approach, initiating and (rapidly) interrupting drugs due to side effects. Drugs are often used in suboptimal dosages to try to avoid negative interactions with concomitant drugs or potentially dangerous systemic side effects, which leads to suboptimal treatment strategies, leaving the patient in pain and inducing a state where the patients will start taking additional (rescue medications) analgesics (often OTC) in an irregular and uncontrolled manner.

Treatment strategies for NeP should therefore be significantly revised, by introducing target-specific treatment options. Such treatment should be focused on the specific sensory syndrome present in the patient, thereby specifically targeting cutaneous disturbances that are present. In this respect, lidocaine 5\% patches can lead to an entirely new and exciting approach to a subset of NeP syndromes. However, the success of such specific treatment probably largely depends on the proper identification of the most 
suitable patients. The purpose of this manuscript is therefore to provide clinicians with the proper tools for identifying such patients, by detailing the appropriate sensory syndromes, and identifying suitable places for the application of the patches and other patient characteristics.

\section{Case report I}

A 74-year-old woman developed a herpes zoster skin rash after the death of her husband. Although she was quickly diagnosed and treated with oral anti-viral medications (acyclovir), she soon developed stabbing and burning pain in the entire area of the skin rash (dermatomes D7 till D9). Analgesic treatment consisting of paracetamol, immediate-release tramadol and long-acting tilidine was initiated but quickly stopped due to pronounced nausea and vomiting. Treatment with pregabaline (150 mg per day) induced dizziness, which was considered as extremely uncomfortable by the patient. Finally, a treatment with lidocaine patches (Versatis $^{\circledR}$; Grünenthal) was initiated. The patient placed 2 patches every day in order to cover the entire painful area. After only 1 week she reported a significant reduction in pain (40\% pain relief compared to pre-application values). After 4 weeks the patient reported a further pain reduction $(-75 \%$ versus pretreatment pain), and an almost completely abolished need for systemic analgesics.

\section{Case report 2}

A 56-year-old man developed an extremely painful syndrome shortly after a surgical procedure to the right foot (microsurgical resection of an interdigital neuroma). Despite multiple pharmacological therapies, consisting of tramadol, low-dose transdermal fentanyl, amitriptyline, gabapentine and venlafaxine, he continued to display severe spontaneous burning pain in addition to a pronounced mechanical hyperalgesia and allodynia among other symptoms. The presence of this NeP syndrome impaired the patient's quality of life in such a way that he had to stop working, refused to wear socks and shoes (moving around on open sandals) and gave up on almost all of his familial and social activities. Daily application of half a lidocaine patch onto the painful skin area quickly reduced the evoked painful symptoms. A short while later the spontaneous pain symptoms also started to decrease. After 8 weeks of treatment, overall pain sensations had diminished by $80 \%$. The patient reported neither local nor systemic side effects, and all other analgesics were stopped. After 12 weeks of treatment he resumed work.

\section{Discussion}

Treatment of NeP has gone through some significant changes in the last decade. Initially, painful neuropathies were treated as any other pain syndromes, merely through analgesics. Then came more specialized anti-neuropathic treatment options, such as $\mathrm{Ca}^{2+}$-channel blockers (gabapentin, pregabalin) and NMDA-receptor antagonists (memantine, ketamine), which led to the appearance of (inter)national guidelines suggesting a multimodal and balanced treatment of NeP (combining analgesics and adjuvants or different classes of adjuvant drugs). This change in pharmaceutical approach to NeP has been accompanied by a growing tendency towards a more mechanism-based treatment. Instead of merely treating the painful symptomatology of etiologies, such as diabetes and herpes zoster infections, physicians have been slowly incorporating underlying pathophysiological mechanisms into the choice of treatment options. As a result, application of combination and multimodal therapies in the management of NeP syndromes has increased.

The introduction of lidocaine-medicated patches should mean an additional and significant step into the transgression from the classical etiology-based approach to NeP to a much more scientifically valid pathophysiology based approach to the treatment of painful neuropathies. A specific therapy for the injured/excited cutaneous nociceptors is now available for the first time. Excited nociceptors are indeed considered a crucial part of the pathophysiology of NeP syndromes..$^{7-10}$ Lidocaine acts through blockade of abnormally functioning (sensitized) Nav 1.7 and Nav $1.8 \mathrm{Na}^{+}$channels in dermal nociceptors, thereby reducing ectopic discharges. ${ }^{11,12}$ Lidocaine has also been shown to regulate T-cell activity and inhibit nitric oxide production, thereby reducing inflammatory processes within the deep tissue, such as injured muscle, joints or constricted nerves. ${ }^{13,14}$ Certain preclinical and clinical findings point towards the existence of additional biological effects, such as blockage of $\mathrm{A} \beta$-afferents conveying allodynia and traveling adjacent to degenerating nociceptors within the affected nerve. ${ }^{15}$ The occurrence of a possible central negative feedback signal can be drawn from the fact that application of lidocaine patches also has been shown to demonstrate an analgesic effect in central NeP syndromes. ${ }^{16}$ The (non)success rate of treatments with the lidocaine $5 \%$ patches will, however, rely heavily on the proper selection of target patients. For this purpose, a clearcut and scientifically valid stepwise approach for identifying appropriate patients is hereby proposed (see also flow-chart diagram, Figure 1). 


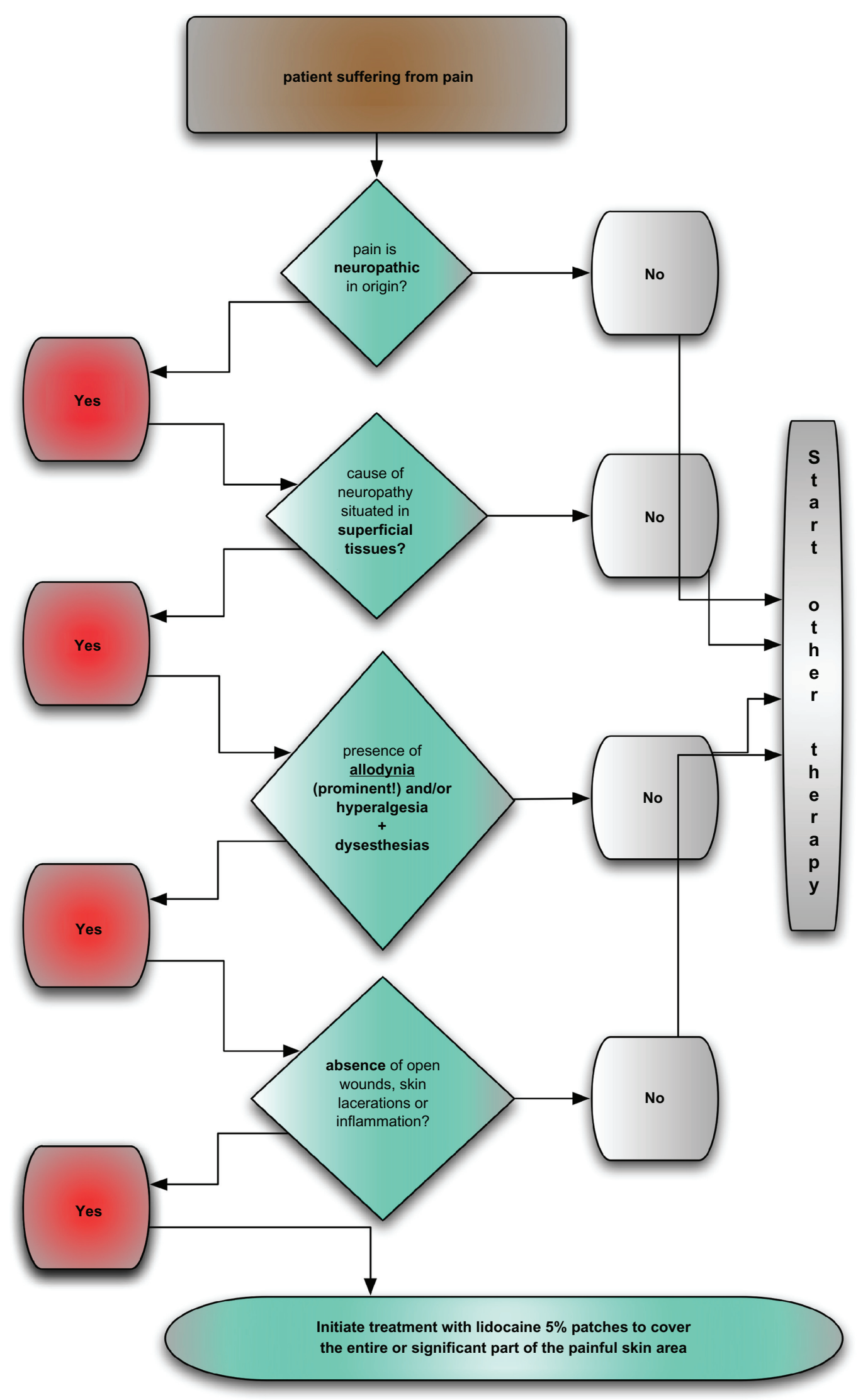

Figure I Flow chart for identifying appropriate patients for treatment with lidocaine medicated patches. 


\section{Who?}

Patients should present with a probable diagnosis of NeP, considering the recently revised definition and grading system. ${ }^{17}$ Although studies have reported analgesic effects of the application of the lidocaine patches in nociceptive (non-neuropathic) pain conditions, ${ }^{18-20}$ the main and most appropriate clinical conditions remain superficial localized painful neuropathies. Patients should present with a clinical history indicative of a NeP syndrome. Neurological examination and ancillary testing should further confirm the existence of a painful neuropathy.

The second, equally important clinical condition is the identification of presence of positive cutaneous sensory disturbances. Recent studies have shown the presence of distinct symptom profiles in patients suffering from NeP. ${ }^{21}$ Patients should have superficial spontaneous pain (dysesthesia). Presence of spontaneous uncomfortable sensory symptoms (paresthesias) should be considered as an additional, but less crucial, clinical feature. In addition to the occurrence of non-evoked pain, presence of hyperalgesia and/or allodynia seems crucial. Allodynic complaints should therefore be the most prominent clinical symptom of the $\mathrm{NeP}$ syndrome in the patient. Additional sensory aberrations can be hyperpathia and temporal or spatial summation. The diagnostic approach to cutaneous NeP syndromes should therefore include the proper identification of positive sensory disturbances through the use of validated (semi-)quantitative sensory testing methods (such as QST).

In a next step, the physician should clearly identify and demarcate the concerned skin area. The physician should ensure that its total area is not too out of proportion in comparison to the size of the lidocaine patches, so that no more than 3 plasters need to be used on a daily basis. In case of small skin areas that are painful it is possible to cut the patches into smaller pieces to cover the painful skin area. Retrospective analysis of use in daily clinical practice has shown a mean value of 0.333 patches per day in our therapies of prolonged duration. ${ }^{22}$ Of course patients should have no previous history of allergic reactions to local anesthetics. Cardiac disease/renal insufficiency and hepatic disorders do not impede the use of lidocaine patches.

\section{Where?}

Proper placement of the patches is the next important clinical feature. Most clinical studies have described the coverage of the entire painful skin area by lidocaine patches. Clinical experience, however, tells us that it is not absolutely necessary to cover the entire painful skin area in order to obtain optimal analgesic results. Coverage of a large part of the painful skin area will in most cases also lead to satisfactory results. It is also proposed that the placement of the patches should be altered each day (for example covering the proximal versus distal part of the painful dermatome). Presence of allodynia can in a small minority of patients lead to exacerbation of pain upon the application of the patch. This allodynic flare-up can be prevented by treating the patient for a short time with systemic anti-neuropathic drugs, such as anti-epileptics.

\section{How?}

Patches should be kept on during 12 to 16 consecutive hours in a day. Initially, the clinical experience was based on a $12 \mathrm{on} / \mathrm{of}$ schedule. This treatment schedule was initially conceived in order to prevent the occurrence of skin irritation well before long-term safety data had become available. Meanwhile, available safety data have indicated that the occurrences of localized skin side effects are minimal with application schemes of 12 to 16 consecutive hours. Patches can be worn during the day or during the night, depending on the exact location of the painful skin area and the adhesive ability of the patch on this particular skin site. Physicians should note, however, that initially patients will probably display some flare up of the pain symptomatology towards the end of the off-period. Over time this time-sensitive course will, however, (rapidly) decrease. During the first period of treatment patients will often report more pronounced analgesia during the on-period (patch in place) and increase of pain after the patch has been removed. This time pattern will change however quickly with continuation of the topical treatment.

\section{Follow-up?}

If the patch successfully decreases pain, analgesic effects should appear rather quickly in a majority of patients. This is in great difference with some systemic treatment options where positive effects can take up to 6 weeks before reaching a maximum value. The beginning of analgesic effects by patch treatment can be expected much sooner, sometimes within a couple of days. It is important to mention that application of lidocaine $5 \%$ patches will not lead to cutaneous anesthesia, since there seems to be only minimal effect on cutaneous $\mathrm{A} \beta$-fibers (continuous release of low concentrations of lidocaine over 12 to 16 hours). This is in contrast with the clear numbness that results from the application of lidocaine cream such as Emla ${ }^{\circledR}$ (AstraZeneca) (release of high concentration over a short period of time). Over time, 
the application of lidocaine patches can be decreased to once every 48 hours, to 3 times a week, and then further, but years of clinical practice has shown that 2 applications a week seem to be the absolute minimum..$^{22}$ In comparison to other anti-NeP therapies, the frequency of application should be decreased gradually, always observing the absence of flare-up before a further reduction is implemented.

\section{Safety aspects?}

A growing body of evidence has indicated that side effects from the use of lidocaine patches are mostly moderate to mild and tend to disappear very quickly after removal of the lidocaine medicated patch. ${ }^{23,24}$ The most common side effect is skin irritation (red discoloration) underneath the patch. This side effect and its severity can easily be diminished by slightly changing the location of the plaster (within the painful dermatome). Systemic side effects are always absent, even during long-term treatment and use of multiple patches on a daily basis. This crucial safety aspect can be explained by the very low systemic absorption of the lidocaine contained in the patches. This unique feature also leads to the absence of interactions with concomitant medications that the patient is taking (eg, cardiovascular medication, cholesterol-lowering drugs, benzodiazepines). The intake of such drugs does not need to be halted, or their doses lowered. There is no risk for occurrence of systemic side effects. It should, however, be stressed that the lidocaine-medicated patches may never be applied to open wounds, mucosa or inflamed tissue in order to avoid increasing the risk of systemic uptake of the lidocaine contained in the patch.

\section{Conclusion}

The introduction of the lidocaine-medicated patches constitutes a milestone development in the treatment of superficial NeP syndromes. Its rapidly occurring analgesic response, low potential for systemic side effects and absence of interaction with concomitant medication make this analgesic agent especially suitable for long-term use in patients prone to side effects of analgesic agents, or in compromised patients with no risk of interactions with concomitant medication. The lidocaine-medicated patch constitutes the first available therapeutic option with a direct effect on the injured or sensitized cutaneous nociceptors. This could make a mechanistic approach to $\mathrm{NeP}$ a clinical reality.

\section{Disclosures}

The authors disclose no conflicts of interest.

\section{References}

1. Attal N, Cruccu G, Haanpaa M, et al. EFNS guidelines on pharmacological treatment of neuropathic pain. Eur J Neurol. 2006;13:1153-1169.

2. Todorovic SM, Jevtovic-Todorovic V. Regulation of T-type calcium channels in the peripheral pain pathway. Channels (Austin). 2007;1:238-245.

3. Romero-Sandoval EA, Horvath RJ, DeLeo JA. Neuroimmune interactions and pain: focus on glial-modulating targets. Curr Opin Investig Drugs. 2008;9:726-734.

4. Aurilio C, Pota V, Pace MC, Passavanti MB, Barbarisi M. Ionic channels and neuropathic pain: physiopathology and applications. $J$ Cell Physiol. 2008;215:8-14.

5. Hans G, Deseure K, Adriaensen H. Endothelin-1-induced pain and hyperalgesia: a review of pathophysiology, clinical manifestations and future therapeutic options. Neuropeptides. 2008;42:119-132.

6. White FA, Jung H, Miller RJ. Chemokines and the pathophysiology of neuropathic pain. Proc Natl Acad Sci U S A. 2007;104:20151-20158.

7. Dina OA, Khasar SG, Alessandri-Haber N, et al. Neurotoxic catecholamine metabolite in nociceptors contributes to painful peripheral neuropathy. Eur J Neurosci. 2008;28:1180-1190.

8. Djouhri L, Koutsikou S, Fang X, McMullan S, Lawson SN. Spontaneous pain, both neuropathic and inflammatory, is related to frequency of spontaneous firing in intact C-fiber nociceptors. J Neurosci. 2006;26:1281-1292.

9. Bostock H, Campero M, Serra J, Ochoa JL. Temperature-dependent double spikes in C-nociceptors of neuropathic pain patients. Brain. 2005; 128:2154-2163.

10. Fields HL, Rowbotham M, Baron R. Postherpetic neuralgia: irritable nociceptors and deafferentation. Neurobiol Dis. 1998;5:209-227.

11. Chevrier P, Vijayaragavan K, Chahine M. Differential modulation of Nav 1.7 and Nav 1.8 peripheral nerve sodium channels by the local anesthetic lidocaine. Br J Pharmacol. 2004;142:576-584.

12. Persaud N, Strichartz GR. Micromolar lidocaine selectively blocks propagating ectopic impulses at a distance from their site of origin. Pain. 2002;99:333-340.

13. Shiga M, Nishina K, Mikawa K, Obara H. The effects of lidocaine on nitric oxide production from an activated murine macrophage cell line. Anesth Analg. 2001;92:128-133.

14. Tanaka A, Minoguchi K, Oda N, et al. Inhibitory effect of lidocaine on T cells from patients with allergic asthma. J Allergy Clin Immunol. 2002;109:485-490.

15. Wasner G, Kleinert A, Binder A, Schattschneider J, Baron R. Postherpetic neuralgia: topical lidocaine is effective in nociceptor-deprived skin. J Neurol. 2005;252:677-686.

16. Hans GH, Robert DN, Van Maldeghem KN. Treatment of an acute severe central neuropathic pain syndrome by topical application of lidocaine 5\% patch: a case report. Spinal Cord. 2008;46:311-313.

17. Treede RD, Jensen TS, Campbell JN, et al. Neuropathic pain: redefinition and a grading system for clinical and research purposes. Neurology. 2008; 70:1630-1635.

18. Kivitz A, Fairfax M, Sheldon EA, et al. Comparison of the effectiveness and tolerability of lidocaine patch $5 \%$ versus celecoxib for osteoarthritisrelated knee pain: post hoc analysis of a 12 week, prospective, randomized, active-controlled, open-label, parallel-group trial in adults. Clin Ther. 2008;30:2366-2377.

19. Gammaitoni AR, Galer BS, Onawola R, Jensen MP, Argoff CE. Lidocaine patch $5 \%$ and its positive impact on pain qualities in osteoarthritis: results of a pilot 2-week, open-label study using the Neuropathic Pain Scale. Curr Med Res Opin. 2004;20 Supp1 2:S13-S19.

20. Burch F, Codding C, Patel N, Sheldon E. Lidocaine patch $5 \%$ improves pain, stiffness, and physical function in osteoarthritis pain patients. A prospective, multicenter, open-label effectiveness trial. Osteoarthritis Cartilage. 2004;12:253-255.

21. Dworkin RH, Jensen MP, Gammaitoni AR, Olaleye DO, Galer BS. Symptom profiles differ in patients with neuropathic versus nonneuropathic pain. J Pain. 2007;8:118-126. 
22. Hans G, Schmidt BL, Strichartz G. Nociceptive sensitization by endothelin-1. Brain Res Rev. 2009;60:36-42.

23. Binder A, Bruxelle J, Rogers P, Hans G, Bosl I, Baron R. Topical 5\% lidocaine (lignocaine) medicated plaster treatment for post-herpetic neuralgia: results of a double-blind, placebo-controlled, multinational efficacy and safety trial. Clin Drug Investig. 2009;29:393-408.
24. Hans G, Sabatowski R, Binder A, Boesl I, Rogers P, Baron R. Efficacy and tolerability of a $5 \%$ lidocaine medicated plaster for the topical treatment of post-herpetic neuralgia: results of a long-term study. Curr Med Res Opin. 2009;25:1295-1305.

\section{Publish your work in this journal}

Clinical Pharmacology: Advances and Applications is an international, peer-reviewed, open access journal publishing original research, reports, reviews and commentaries on all areas of drug experience in humans. The manuscript management system is completely online and includes a very quick and fair peer-review system, which is all easy to use.
Visit http://www.dovepress.com/testimonials.php to read real quotes from published authors. 